\title{
Silent Sinus Syndrome- A Blowout Fracturo Mimic
}

\section{Chanabasappa Chavadi', Swetha Kory ${ }^{2}$, Ajit Mahale', Merwyn Fernandes'}

From the Department of Radiodiagnosis, Kasturba Medical College', Mangalore;

Yenepoya Medical College ${ }^{2}$, Mangalore, India.

\begin{abstract}
:
The silent sinus syndrome, also known as imploding antrum and chronic maxillary sinus atelectasis, consists of painless enophthalmos, facial asymmetry with inward retraction of the ipsilateral maxillary sinus walls as seen on imaging studies. It is a well-recognized entity in the field of otolaryngology, but the syndrome remains relatively unknown among radiologists despite its characteristic radiological features. The chronic maxillary sinus atelectasis presenting to the Emergency Room with facial trauma, this entity may, to the radiologist unaware of its existence, be misdiagnosed as an orbital floor blowout fracture, especially when the trauma is ipsilateral. Features that distinguish it from the blow-out fracture are the associated deformities of the other maxillary walls, total opacification of the sinus (as opposed to an air-fluid level), and osteolysis of the sinus walls (if present). It can be definitively treated by creating an outlet for mucous drainage from the obstructed sinus, the goal of treatment being to arrest disease progression without development of further deformity. The purpose of this article is to acquaint the reader with the clinical and imaging features of the silent sinus syndrome.
\end{abstract}

Key words: Hyperplasia, Enophthalmos, Facial Asymmetry, Orbital Fractures, Paranasal sinuses.

\section{Introduction}

Silent sinus syndrome (SSS) constitutes unilateral spontaneous enophthalmos due to increased orbital volume and retraction of the orbital floor [1]. Retraction of the ipsilateral maxillary sinus walls, seen on imaging can be mistaken for an old fracture deformity. Although initial evaluation of this condition is made by the ophthalmologist or otolaryngologist, it has to be confirmed by imaging. The article proposes to familiarize the reader with the imaging features, etio-pathogenesis and clinical relevance for early detection and treatment. A case of silent sinus syndrome, diagnosed incidentally in a case of facial trauma, is being reported here.

\section{Case Report}

A 30-year-old man presented to the emergency services with history of facial injury. Incidental note was made of retracted right upper eyelid when compared with the left. He experienced no other

Corresponding Author: Dr. Chanabasappa Chavadi

Email: chavadidoc@gmail.com

Received: January 12,2014 | Accepted: January 25, 2014 | Published Online: February 15, 2014 This is an Open Access article distributed under the terms of the Creative Commons Attribution License (creativecommons.org/licenses/by/3.0)

Conflict of interest: None declared | Source of funding: Nil | DOl: http://dx.doi.org/10.17659/01.2014.0016 
eye symptoms and reported no history of old facial trauma. No history related to sinusitis, recurrent upper respiratory tract infection or prior surgery for paranasal sinuses could be elicited. Physical examination revealed a deep right upper eyelid sulcus and enophthalmos of $2 \mathrm{~mm}$. The eye was also depressed and displaced medially. There was no diplopia, eye movements and vision were however normal. There were normal sized tonsils with no evidence of inflammation in pharynx. His thyroid function test, namely TSH, T3 and T4 were normal. CT scan of the face was performed without intravenous contrast, which revealed depressed right orbital floor with retraction of the walls of maxillary sinus into the sinus lumen [Fig.1] and atelectatic opacified right maxillary sinus. The uncinate process was opposed to the inferomedial orbial margin with consequent narrowing of the ostia of ostiomeatal complex [Fig.1]. There was inward retraction of all the sinus walls with occlusion of the maxillary infundibulum and enlarged adjacent middle meatus [Axial and coronal image in Fig.2]. There was no destruction of the bony walls of sinus or extension

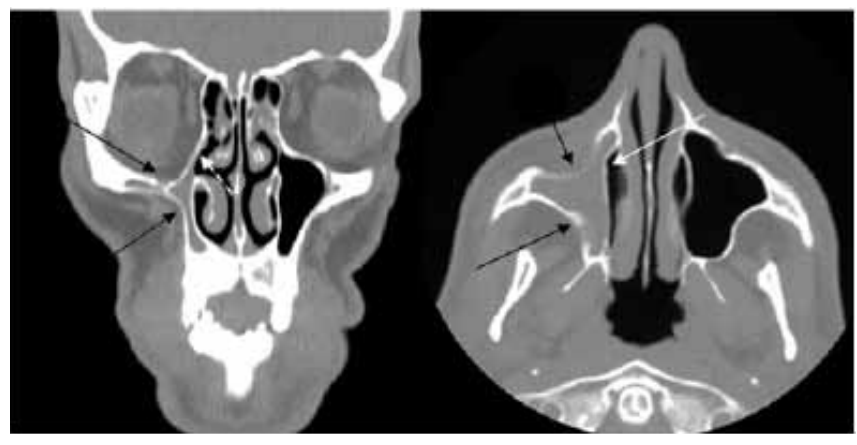

Fig.1: Unenhanced coronal and axial image illustrates the depressed orbital floor, retraction of the walls of maxillary sinus into the sinus lumen (black arrows). Uncinate process is opposed to the inferomedial orbital rim (white arrow in coronal image) occluding the ostia of right ostiomeatal complex. Narrow infundibulum and atelectiatic opacified right maxillary sinus are also seen. of disease process to soft tissues of the facial region. The downward retraction of the orbital floor created asymmetry of eye. There was no evidence of fracture or hemosinus. Orbital or paranasal sinus neoplasm was excluded. There was no deviation of the nasal septum and other paranasal sinuses were normally pneumatised. Based on imaging findings a diagnosis of 'silent sinus syndrome' was arrived at. Silent sinus syndrome or chronic maxillary sinus atelectasis is a rare condition that can pose a diagnostic challenge, as the medical history is often noncontributory.

\section{Discussion}

The patient with SSS may present with unilateral ptosis or retraction of upper eyelid, a deep superior sulcus or orbital asymmetry. Enophthalmos is usually seen on physical examination. Other physical signs would include hypoglobus, upper-lid retraction, deepened upper-lid sulcus, and malar depression. This occurs because of atelectasis of the ipsilateral maxillary sinus and if the condition is left untreated, may result in complete obliteration of the sinus with worsening enophthalmos and hypoglobus.

Etiology is usually idiopathic [2]. The most popular theory of pathogenesis is that ostiomeatal

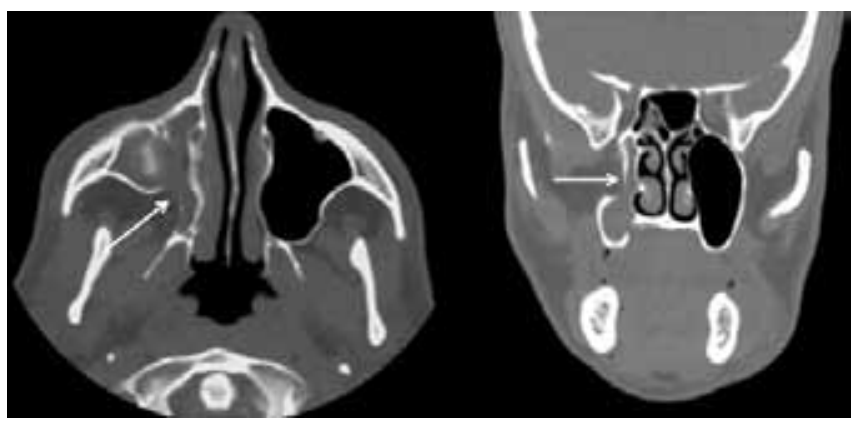

Fig.2: Axial image at more superior level and coronal at more posterior level also illustrates the retraction of maxillary sinus walls. Normal aerated left maxillary sinus is noted. 
obstruction results in reduced aeration of the antrum, causing negative sinus pressure and atelectasis $[1,3]$. Supporting this hypothesis, in our case there is uncinate process being opposed to the inferomedial orbital rim [white arrow in coronal Fig-1] occluding the ostia of right ostiomeatal complex. This is similar to middle ear atelectasis secondary to eustachian tube dysfunction. Low-grade inflammatory response that sets in due to stagnant mucus within the sinus, incites a osteolysis of the sinus walls. The weakened sinus walls are pulled into the sinus lumen by the negative sinus pressure. Damage to the osteomeatal complex after endoscopic sinus surgery is also postulated as the causative factor [4]. Conversely, there are instances of relatively acute onset of symptoms occurring after orbital decompression, where it is appropriately described as "imploding antrum syndrome" [5]. The other theory involves hypoplasia. It assumes that SSS is the result of infection in the congenitally hypoplasic maxillary sinus. This may represent a less severe form of the silent sinus syndrome, with the smaller sinus volume and shorter thicker sinus walls affording the sinus protection against osteolysis and negative pressure effects. Acute onset enophthalmos is commonly seen after major orbital floor fractures. Cases of late onset enophthalmos after minor trauma have been reported leading to contraction of the maxillary sinus, here it developed years after trauma suggesting protracted natural course of silent sinus syndrome after ostiomeatal obstruction [6].

The SSS can be definitively treated by creating a nasal antral window for mucous drainage from the obstructed sinus endoscopically [7]. The orbital floor repair is usually performed in patients with diplopia or severe cosmetic deformity and in patients with little improvement after functional endoscopic sinus surgery. Implantation of an alloplastic orbital floor graft is usually done to improve deformity in a case of late enophthalmos mimicking 'silent sinus syndrome' secondary to orbital trauma [8]. Transconjunctival approach for orbital floor repair along with Caldwell-Luc surgery is also proposed [9].

We conclude that the silent sinus syndrome consists of commonly insidious onset of painless facial asymmetry and enophthalmos caused by chronic maxillary sinus atelectasis and in patients presenting to the emergency room with facial trauma, this entity may, to the radiologist unaware of its existence, be misdiagnosed as an orbital floor blowout fracture, especially when the trauma is ipsilateral. Features that distinguish it from the blow-out fracture are the associated deformities of the other maxillary walls, total opacification of the sinus (as opposed to an air-fluid level), and osteolysis of the sinus walls (if present). Although the diagnosis is usually suspected clinically, it is confirmed radiologically and can be definitively treated by creating an outlet for mucous drainage from the obstructed sinus, the goal of treatment being to arrest disease progression without development of further deformity.

\section{References}

1. Soparkar CN, Patrinely JR, Davidson JK. Silent sinus syndrome: New perspectives. Ophthalmology. 2004;111:414-415.

2. Anna Illner, Christian Davidson $H$, Harnsberger HR, Hoffman J. 'Silent sinus syndrome-clinical and radiographic findings. Am J Roental. 2002;178:503-506.

3. Gillman GS, Schaitkin BM, May M. Asymptomaticenophthalmos: the silent sinus syndrome. Am J Rhinol. 1999; 13:459-462.

4. Levine SB, Mitra S. Maxillary sinus involution after endoscopic sinus surgery in a child: a case report. Am J Rhinol. 2000;14:7-11.

5. Rose GE, Sandy C, Hallberg L, Moseley I. Clinical and radiologic characteristics of the Imploding antrum or "Silent sinus syndrome". Ophthalmology. 2003;1 10:811-818.

6. Montezuma SR, Gopal H, Savar A, Turalba A, Cestari DM, Torun N. "Silent sinus syndrome presenting as enophthalmos long after orbital 
trauma". J Neuro-Ophthal. 2008;28:107-1 10.

7. Amin R Javer, Elaheh Akbari, Jamil Manji, Ronak Rahmanian, San Sunkarneni. The Efficacy of Endoscopic Sinus Surgery in the Management of Silent Sinus Syndrome. Otolaryngol Head Neck Surg. $2011 ; 145(2): 267$.

8. Ross Jason J, Kersten Robert C. Late Enopthalmos
Mimicking Silent Sinus syndrome secondary to orbital trauma. Journal of Craniofacial Surgery. 2005; 1 6(5):840-843.

9. Dailey RA, Cohen Jl. Surgical repair of the silent sinus syndrome. Ophthal Plast Reconstr Surg. 1995; $11(4): 261-268$. 